\title{
Conditional Covariance-Based Subtest Selection for Polytomous DIMTEST
}

\author{
Tan $\mathrm{Li}^{1}$, Brian Habing ${ }^{2}$, and Louis Roussos ${ }^{3}$
}

\begin{abstract}
Dimensionality assessment is an important component of test validation. While the vast majority of statewide standardized tests contain both dichotomous and polytomous items, much of the work in dimensionality assessment has focused on the case of dichotomous item exams. Poly-NEWDIM is a polytomous version of DIMTEST that is a nonparametric hypothesis testing procedure for dimensionality assessments based on comparison of an assessment subtest (AT) with a partitioning subtest (PT). A good AT and PT selection is vital to the performance of Poly-NEWDIM. The proposed two new AT-selection procedures, HCA/CCPROXPolyDIMTEST (HCP) and HCA/CCPROX-PolyNEWDIM $(\mathrm{HCN})$, perform better than the previous AT-selection method (HCD) in terms of power. Moreover, $\mathrm{HCN}$ shows less sensitivity than HCP to sample size, correlation, and structural complexity. The results also indicate that $70 \%$ of data for AT selection is appropriate for all three kinds of tests (dichotomous only, polytomous only, and mixed) with large sample sizes and dichotomous tests with small sample sizes, while $50 \%$ is good for polytomous tests and mixed tests with small sample sizes.
\end{abstract}

\section{Keywords}

dimensionality, DIMTEST, polytomous items

\section{Introduction}

Many of the common item response theory (IRT)-based procedures entail three assumptions: monotonicity $(\mathrm{M})$, local independence $(\mathrm{LI})$, and unidimensionality $(d=1)$. Monotonicity states that for any possible score, $k>0$, the probability that an examinee's score $U_{i}$ for item $i$ is higher than $k, P\left[U_{i} \geq k \mid \Theta=\theta\right]$, increases as the examinee's ability $(\theta)$ increases; local independence, also known as conditional independence, assumes that the responses to test items are independent of each other, given the value of $\theta$; and unidimensionality assumes that all items in an exam measure the same $\theta . \mathrm{M}, \mathrm{LI}$, and $d=1$ are often assumed to hold in the case of educational

\footnotetext{
'Florida International University, Miami, FL, USA

${ }^{2}$ University of South Carolina, Columbia, SC, USA

${ }^{3}$ Measured Progress, Dover, NH, USA
}

\section{Corresponding Author:}

Tan Li, Department of Biostatistics, Robert Stempel College of Public Health and Social Work, Florida International University, II 200 S.W. 8th Street, AHC5-464, Miami, FL 33199, USA.

Email: tanli@fiu.edu 
assessments. These assumptions underlie most of the estimation, differential item functioning detection, and equating procedures used in practice.

However, the assumption of unidimensionality is often not reasonable. For example, a statistics exam using context of real-life problems would presumably measure statistics ability as well as reading ability. Violating the unidimensionality assumption could seriously bias item and ability parameter estimation (e.g., Ackerman, 1989; Kirisci \& Hsu, 1995). Thus, it is important that an evaluation of the test's dimensionality be efficiently and effectively carried out on a routine basis. Such routine testing is substantially expedited by the use of a reliable hypothesis test. If a reliable hypothesis test results in nonrejection, practitioners can safely forego timeconsuming detailed analyses that attempt to describe and explain the multidimensionality. Such analyses can be confined to only those tests that show strong evidence of multidimensionality. Hattie (1985) provides a summary of various procedures up to that time, and notes in particular that classic linear factor analysis (raw correlation or tetrachorics) performs poorly for dichotomous items. Various other dimensionality assessment procedures have since been developed (Reckase, 2009; Tate, 2003).

The DIMTEST procedure (Nandakumar, Yu, Li, \& Stout, 1998; Stout, 1987; Stout, Froelich, $\&$ Gao, 2001) is often used to test the null hypothesis that an exam is locally independent and unidimensional. It does this by dividing the test to two subtests (an assessment subtest called AT and a partitioning subtest called PT) and testing whether there are any local dependencies among the AT items, conditioned on the score on the partitioning test. DIMTEST has been widely studied for dichotomous item exams, and has good power when AT and PT are chosen well (e.g., Froelich \& Habing, 2008). If AT and PT are chosen poorly (e.g., both are random samples of items), the procedure will have power near 0 .

Polytomous items are increasingly used in large-scale standardized tests. The original PolyDIMTEST (Li \& Stout, 1995) was an adaptation of DIMTEST (Stout, 1987) to polytomous items. It requires the use of a second subtest called AT2 to correct bias, and is much less effective than the bootstrap bias correction used in the latest version of dichotomous DIMTEST (Stout et al., 2001). Poly-DIMTEST without AT2 (Li et al., 2010; Li, 2011) applies the bootstrap procedure to the original Poly-DIMTEST for bias correction. NEWDIM (Seo \& Roussos, 2009, 2010) improved Stout, Froelich, and Gao's procedure using a different formulation of the statistics that they argued was theoretically more justified and mathematically simpler. This included weighting the sample conditional covariances to better approximate the true average of the conditional covariances. Poly-NEWDIM applies this change to improve Poly-DIMTEST without AT2, and demonstrates closer to nominal Type I error and better power (Li et al., 2010; Li, 2011). Similar to DIMTEST, the selection of AT and PT subtests decides the performance of Poly-NEWDIM.

Usually the AT and PT selection is decided either by subject area experts or by an exploratory statistical procedure. In the latter case, the examinees are randomly divided into two groups (as in cross-validation). One group is used to select the AT and PT subtests, and the other is used to calculate the DIMTEST statistic. The original Poly-DIMTEST procedure was packaged with a linear factor analysis to choose AT. However, linear factor analysis performs poorly, especially when the ability distribution of examinees was different from the distribution of the dichotomous item difficulty parameters (e.g., Hattie, 1985; Seraphine, 2000). The poor performance is reinforced when using it to select dimensionally distinct clusters (Froelich \& Habing, 2008; Hattie, Krakowski, Rogers, \& Swaminathan, 1996).

Froelich and Habing (2008) developed a method of AT subtest selection (HCD) that combines the methods of HCA/CCPROX (Roussos, Stout, \& Marden, 1998) and DETECT (Kim, 1994; Zhang \& Stout, 1999b), and results in good power for DIMTEST. However, the HCD method performs poorly in conjunction with Poly-NEWDIM either when the correlations are 
high between two latent abilities measured in a two-dimensional exam, or when there are only a few items measuring one of the two latent abilities (Li et al., 2010). Thus, the purpose of this article was to develop two new AT-selection methods based on the test statistics of PolyDIMTEST without AT2 and Poly-NEWDIM, and compare them with HCD using Type I error and power simulation studies.

The rest of this article is organized as follows: The section "Poly-NEWDIM and ATSelection Methods" describes the details of HCD and the proposed AT-selection methods. The section "Appropriate Percentage for AT Selection" investigates the appropriate percentage of examinees used for AT selection and compares the performances of these AT-selection methods in conjunction with Poly-NEWDIM, based on simulation study. The section "Concluding Remarks" gives concluding remarks. Part of this work appears in Li, Habing, \& Roussos's (2011) study.

\section{Poly-NEWDIM and AT-Selection Methods}

To illustrate how the methods proposed in this study work, conditional covariance theory and the Poly-NEWDIM procedure are first briefly introduced using a simple exam as an example.

Figure 1 geometrically shows a four-item two-dimensional exam. Assuming that the two latent abilities $\left(\theta_{1}\right.$ and $\left.\theta_{2}\right)$ are compensatory for the items on the test (higher levels of one ability are able to compensate for lower levels on the other one), the direction of each item can be represented by the direction of its discrimination parameter vector (Ackerman, 1996; Reckase, 1985; Reckase \& McKinley, 1991). Furthermore, the direction of the linear ability composite $\Theta_{T}$ best measured by this exam can be represented by a weighted average of all the item discrimination parameter vectors with weights determined by maximizing the expected multidimensional critical ratio function, which gives the average discriminating power of the exam (Zhang \& Stout, 1999a).

In conditional covariance theory, Zhang et al. (1999a, 1999b) and Zhang (2007) assume that the exam follows a generalized compensatory model. The multidimensional three parameter logistic (M3PL) model (Reckase, 1997) for dichotomous items is one example of this:

$$
P\left[U_{i}=1 \mid \boldsymbol{\Theta}=\theta\right]=c_{i}+\frac{1-c_{i}}{1+e^{-1.7\left(a_{i}^{T} \theta-\left|a_{i}\right| b_{i}\right)}},
$$

where $U_{i}$ is the response to item $i, \mathbf{a}_{i}=\left(a_{i 1}, a_{i 2}, \ldots, a_{i m}\right)^{T}$ is the discrimination parameter vector, $b_{i}$ is the difficulty parameter, and $c_{i}$ is the guessing parameter. The $\mathbf{a}_{i}^{T} \theta$ implies how higher levels of ability on some dimensions are able to compensate for lower levels of others. In the twodimensional case, conditional covariance theory says that an item pair on the same side of $\Theta_{T}$ will have a positive conditional covariance, and that an item pair on opposite sides will have a negative conditional covariance. Hence, in Figure 1, Items 1 and 2 will have a positive conditional covariance, Item Pairs $(1,4)$ and $(2,4)$ will have negative conditional covariances, and any pair with Item 3 will have a zero conditional covariance. The signs and magnitudes of these covariances are then used to construct various exploratory and inferential procedures (e.g., Stout et al., 1996). In higher dimensions, the result is based on the angle $\gamma_{i l}$ between the projections of the two discrimination parameter vectors on the hyperplane $\Theta_{T}^{\perp}$ which is $(d-1)$ dimensional and orthogonal to $\Theta_{T}$ (Zhang et al., 1999a).

The basic idea behind Poly-NEWDIM (Li et al., 2010; Li, 2011) is to divide a test into two subtests: an AT and a PT. Ideally, if the exam is potentially multidimensional, AT and PT will be selected, so that the AT items are dimensionally homogeneous and distinct from the PT items (see Figure 2). The hypotheses tested are as follows: 


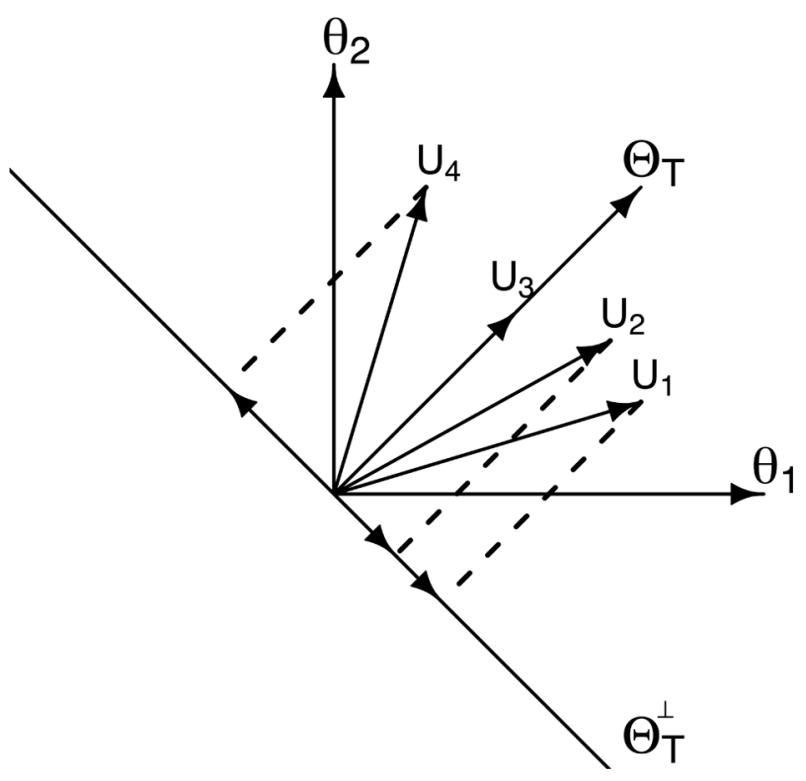

Figure I. Graphical representation of multidimensional test items.

$$
\mathrm{H}_{0}: \Theta_{\mathrm{AT}}=\Theta_{\mathrm{PT}} \quad \text { versus } \quad \mathrm{H}_{1}: \Theta_{\mathrm{AT}} \neq \Theta_{\mathrm{PT}}
$$

That is, the null hypothesis is that the AT and PT subtests measure the same dimension (i.e., the test is unidimensional), and the alternative hypothesis is that AT is measuring a different dimension than the remainder of the test (PT). The parameter $T$ is defined as

$$
T=\sum_{i \neq l \in \mathrm{AT}} \int_{-\infty}^{\infty} \operatorname{Cov}\left(U_{i}, U_{l} \mid \Theta_{\mathrm{PT}}=\theta_{\mathrm{PT}}\right) f\left(\theta_{\mathrm{PT}}\right) d \theta_{\mathrm{PT}}
$$

where $U_{i}$ and $U_{l}$ are responses to item $i$ and item $l$, respectively, in AT, and $f(\theta)$ is the density function of $\Theta_{T}$. Under the null hypothesis, $\operatorname{Cov}\left(U_{i}, U_{l} \mid \Theta_{\mathrm{PT}}=\theta_{\mathrm{PT}}\right)$ is 0 for any pair of items $i$ and $l$ in AT as AT and PT measure the same dimension. Therefore, when the null hypothesis is true, $T$ is equal to 0 . For a two-dimensional exam, $T$ is positive with a good AT selection, as shown in Figure 2, according to the conditional covariance theory. Under the null hypothesis, the standardized $\hat{T}, \hat{T}^{*}=\frac{\hat{T}}{\hat{\sigma}_{T}}$, asymptotically follows a standard normal distribution. The null hypothesis is rejected at significance level of $\alpha$ when $T^{*}$ is larger than the $(1-\alpha)$ percentile of the standard normal distribution.

In practice, it is necessary to estimate the conditioning ability $\Theta_{P T}$ to calculate $T$ as $\Theta_{P T}$ is a latent variable. In the Poly-NEWDIM procedure as well as other DIMTEST procedures, the observed sum score of PT, $S_{\mathrm{PT}}$, is used as an estimator of $\Theta_{\mathrm{PT}}$, which leads to a positive bias on $\operatorname{Cov}\left(U_{i}, U_{l} \mid \Theta_{\mathrm{PT}}\right), i \neq l \in \mathrm{AT}$. This is because under the null hypothesis, items $i$ and $l$ provide extra information about $\Theta_{\mathrm{PT}}$ beyond what $S_{\mathrm{PT}}$ does. Answering item $i$ correctly means that the estimate of $\Theta_{\mathrm{PT}}$ should be increased, which in turn means that the examinee has a better chance of getting item $l$ correct. Poly-NEWDIM employs a nonparametric IRT-based parametric bootstrap procedure (e.g., Habing, 2001; Kim, 1994; Stout et al., 2001) to correct for this bias. 


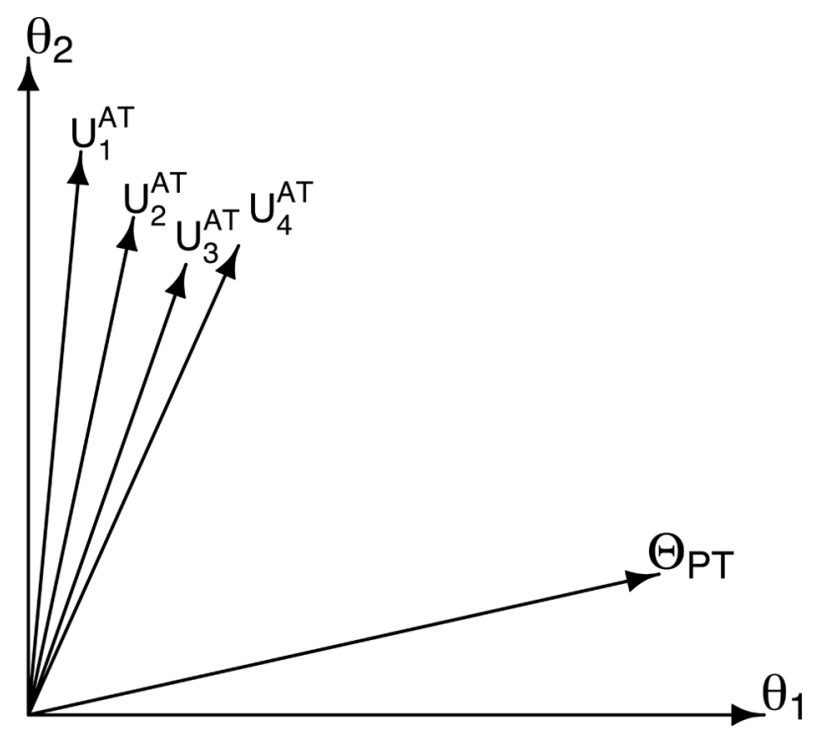

Figure 2. An example of good subtest selection.

Note. AT = assessment subtest; $\mathrm{PT}=$ partitioning subtest.

As mentioned earlier, a good AT and PT selection is one such that the AT items are dimensionally homogeneous and distinct from the PT items (see Figure 2). If the selection is poor (Figure 3), the AT items will be on both sides of the test direction of the PT $\left(\Theta_{\mathrm{PT}}\right)$, and some conditional covariances will be positive, some will be negative, and some will be near 0 . The test statistic will then be small, which will lead to a failure to reject the unidimensionality hypothesis when the test is actually multidimensional.

\section{HCA/CCPROX-DETECT AT Selection}

In response to the poor performance of linear factor analysis in selecting the AT subtest, Froelich and Habing (2008) proposed a subtest selection method for dichotomous item DIMTEST based on HCA/CCPROX (Roussos et al., 1998) and DETECT (Kim, 1994; Zhang \& Stout, 1999b). The HCA/CCPROX-DETECT AT-selection method (HCD) has two steps:

Step 1. Perform the HCA/CCPROX cluster analysis on the test data to provide potential AT/ PT pairs.

Step 2. Calculate the DETECT index on each of the AT/PT pairs found in Step 1. The DETECT statistic is given by

$$
D\left(\mathcal{P}, \Theta_{T}\right)=\frac{2}{n(n-1)} \sum_{1 \leqslant i<l \leqslant n} \delta_{i l} E\left[\operatorname{Cov}\left(U_{i}, U_{l} \mid \Theta_{T}\right)\right],
$$

where $P$ is any partition of an exam, $n$ is the number of items in the exam, and 


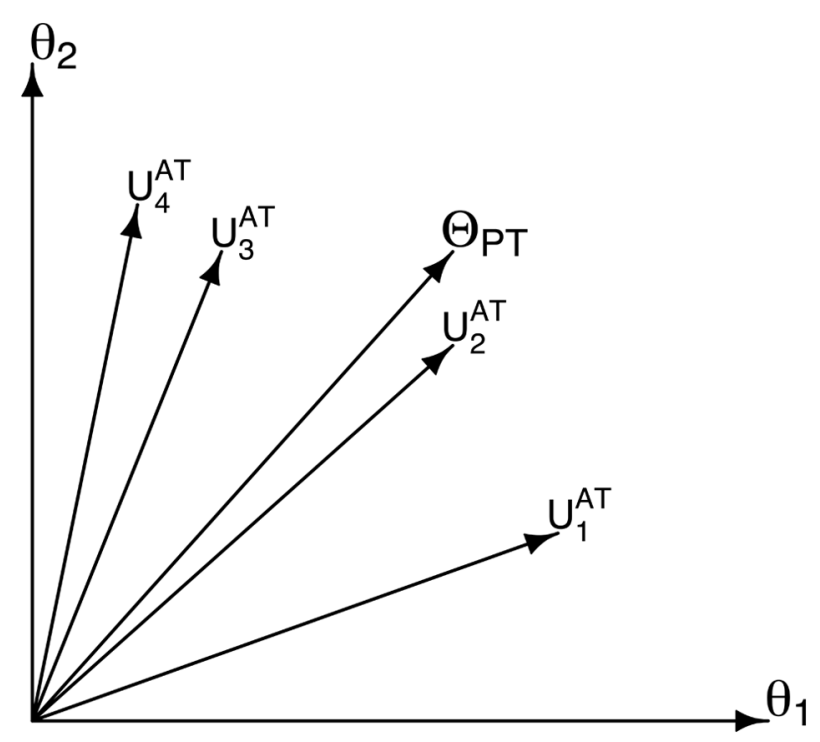

Figure 3. An example of bad subtest selection.

Note. AT = assessment subtest; $\mathrm{PT}=$ partitioning subtest.

$$
\delta_{i l}=\left\{\begin{array}{cc}
+1, & \text { if item } i \text { and item } l \text { are within the same cluster; } \\
-1, & \text { otherwise, }
\end{array}\right.
$$

is maximized when all of the items fall into separate clusters as defined by the conditional covariance theory. Select the AT/PT pair with the maximum DETECT index.

In Step 1, HCA/CCPROX groups the items into progressively larger clusters based on their dimensional similarity. All of the items are separated, and then the two with the most positive conditional covariance are clustered together. The various clusters continue to join, until in the final step all of the items are in a single cluster. Each of the clusters that has between 2.5 of the items is taken as a potential AT subtest, with the remaining items being the corresponding PT subtest. This makes the items in the potential AT most dimensionally similar. Note that a minimum two items are used for the smallest potential AT subtest due to the changes using polytomous items caused to the simulation design; Froelich and Habing (2008) used four items as the smallest one. In Step 2, the maximized DETECT index makes sure that AT and PT are dimensionally distinct. Therefore, the selected partition will satisfy the requirements of a good AT/PT selection.

\section{HCA/CCPROX-PolyDIMTEST (HCP) Selection and HCA/CCPROX-PolyNEWDIM (HCN) Selection}

Although the HCD method of selecting subtests performs well for dichotomous items, Li et al. (2010) found that it does not perform as well for polytomous items. Instead of using DETECT index to decide the optimal AT/PT pair, the test statistics of Poly-DIMTEST without AT2 and Poly-NEWDIM can also be used to select the best AT/PT pair from the cluster solutions of HCA/CCPROX. These choices should be good as they are based on the statistic actually being 
used by DIMTEST instead of the DETECT index (as is done by HCD). Similarly to HCD, the HCP and HCN methods also follow two steps.

\section{$H C P$}

Step 1. Same as HCD.

Step 2. Calculate the HCP index, $H_{P}=\hat{T}^{*}-\bar{T}_{B}^{*}$, for each potential AT/PT pair. The pair with greatest index is selected as AT and PT.

$H_{P}$ is the numerator of $T_{p d}=\frac{\hat{T}^{*}-\bar{T}_{B}^{*}}{\sqrt{1+1 / N}}$, the test statistic for Poly-DIMTEST without AT2, where $\hat{T}^{*}$ is the estimated standardized $T$ in Equation 3 using a complex formula for the asymptotic variance estimator (see details in Stout et al., 2001), $N$ is the number of samples in bootstrap procedure, and $\bar{T}_{B}^{*}$ is the average of $\hat{T}^{*}$ for the $N$ bootstrapped sample. The denominator is removed for $H_{P}$ as it is a constant and will not affect the AT selection.

\section{$\mathrm{HCN}$}

Step 1. Same as HCD.

Step 2. Calculate the HCN index, $H_{N}=\frac{\hat{T}-\bar{T}_{B}^{*}}{\hat{\sigma}_{T}}$, for each potential AT/PT pair. The pair with greatest index is selected as AT and PT.

$H_{N}$ is part of $T_{p n d}=\frac{\hat{T}-\bar{T}_{B}}{\hat{\sigma}_{T} \sqrt{1+1 / N}}$, the test statistic for Poly-NEWDIM. Similar to HCP, the constant $\sqrt{(1+1 / N)}$ is removed. In Poly-NEWDIM, $\hat{T}$ estimates $T$ in Equation 3 using an alternate way to weight the conditional covariances than HCP, and using $\hat{\sigma}_{T}$, the standard deviation of $\hat{T}$ for the $N$ bootstrapped samples, instead of the complex asymptotic variance estimator for HCP (see details in Li et al., 2010; Li, 2011).

HCA/CCPROX in Step 1 makes the items in the potential AT dimensionally similar. The maximized $H_{P}$ and $H_{N}$ in Step 2 make sure that AT and PT are dimensionally distinct, as $\hat{T}^{*}$ and $\hat{T}$ in the formulas are positive when AT and PT are measuring different dimensions, and are 0 when $\mathrm{AT}$ and $\mathrm{PT}$ are measuring the same dimension.

\section{Monte Carlo Simulation Study}

Both a Type I error study and a power study were employed to decide the appropriate percentage of data used for AT selection (with the rest used to perform Poly-NEWDIM) and to evaluate the performances of the AT-selection methods in conjunction with Poly-NEWDIM.

For the Type I error study, the three-parameter logistic model (Birnbaum, 1968)

$$
P\left(U_{i j}=1 \mid \Theta=\theta_{j}\right)=c_{i}+\frac{1-c_{i}}{1+e^{-1.7 a_{i}\left(\theta_{j}-b_{i}\right)}},
$$

and graded response model (GRM; Samejima, 1997)

$$
P\left(U_{i j}=g \mid \Theta=\theta_{j}\right)=\frac{1}{1+e^{-1.7 a_{i}\left(\theta_{j}-b_{i}+d_{i, g-1}\right)}}-\frac{1}{1+e^{-1.7 a_{i}\left(\theta_{j}-b_{i}+d_{i, g}\right)}},
$$

were used to simulate unidimensional test datasets. Here, $a_{i}$ is the discrimination parameter, $b_{i}$ is the difficulty parameter, and $c_{i}$ is the guessing parameter for item $i, d_{i, g}$ is a category 
Table I. 3PL Parameter Distributions.

\begin{tabular}{cl}
\hline Parameters & Distribution \\
\hline$a_{i}$ & $\log N(-0.14,0.35)$ \\
$b_{i}$ & $N(-0.23,0.95)$ \\
$c_{i}$ & $\log N(-1.63,0.45)$ \\
\hline
\end{tabular}

Note. $3 \mathrm{PL}=$ three-parameter logistic.

Table 2. GRM Parameter Values.

\begin{tabular}{lllllllll}
\hline$\mu_{d_{i, 1}}$ & $\sigma_{d_{i, 1}}$ & $\mu_{d_{i, 2}}$ & $\sigma_{d_{i, 2}}$ & $\mu_{d_{i, 3}}$ & $\sigma_{d_{i, 3}}$ & $\rho_{12}$ & $\rho_{13}$ & $\rho_{23}$ \\
\hline 1.3238 & .5431 & .3398 & .2542 & -.3592 & .2738 & .6145 & -.6907 & -.6428 \\
\hline
\end{tabular}

Note. GRM = graded response model.

parameter for $g=1,2, \ldots, m_{i}$ with constraints $\sum_{g=1}^{m_{i}} d_{i, g}=0$ and $d_{1}>d_{2}>\ldots>d_{m_{i}}\left(m_{i}=4\right.$ in this research).

The generated distributions for the item parameters used for the simulation study are estimated from real data based on a large multi-year pool of 729 Grade 5 math items, $12 \%$ of which were polytomous items (see Tables 1 and 2).

The $j^{\text {th }}$ examinee's ability $\theta_{j}$ was randomly generated from $N(0,1)$. Both a moderately long test (52 points) and a medium length test (32 points) were employed. To study the effect of multiple choice (MC, dichotomous item)-constructed response (CR, polytomous item) composition, four mixtures of $\mathrm{MC}$ and $\mathrm{CR}$ items were employed: $100 \% \mathrm{MC}$ items, $85 \% / 15 \% \mathrm{MC} / \mathrm{CR}, 50 \% /$ $50 \% \mathrm{MC} / \mathrm{CR}$, and $100 \% \mathrm{CR}$. To study the effect of sample size, the number of simulated examinees was 500 or 1,000 . The total number of different combinations of factors was 2 exams $\times 4$ compositions $\times 2$ sample sizes $=16$ for $\mathrm{HCD}, \mathrm{HCP}$, and $\mathrm{HCN}$, respectively.

For the power study, two-dimensional 3PL model (Equation 8) and GRM model (Equation 9) were used to simulate two-dimensional test datasets. The distributions and values of all the parameters were the same as in Tables 1 and 2 . The $j^{\text {th }}$ examinees' ability parameters $\left(\theta_{1 j}, \theta_{2 j}\right)$ were randomly generated for each examinee from a standard bivariate normal distribution with a specified correlation $(.6, .7$, or .8$)$ between the dimensions. Larger sample sizes and lower correlations were not studied as the power was already generally high for 1,000 examinees and/or correlations of .6.

$$
\begin{aligned}
P\left(U_{i j}=1 \mid \theta_{1 j}, \theta_{2 j}\right) & =c_{i}+\frac{1-c_{i}}{1+e^{-1.7\left(a_{i 1} \theta_{1 j}+a_{i 2} \theta_{2 j}-b_{i} \sqrt{a_{i 1}^{2}+a_{i 2}^{2}}\right)}}, \\
P\left(U_{i j}=g \mid \theta_{1 j}, \theta_{2 j}\right) & =\frac{1}{1+e^{-1.7\left[a_{i 1} \theta_{1 j}+a_{i 2} \theta_{2 j}-\left(a_{i 1}+a_{i 2}\right)\left(b_{i}-d_{i, g-1}\right)\right]}} \\
& -\frac{1}{1+e^{-1.7\left[a_{i 1} \theta_{1 j}+a_{i 2} \theta_{2 j}-\left(a_{i 1}+a_{i 2}\right)\left(b_{i}-d_{i, g}\right)\right]}} .
\end{aligned}
$$

Simple structure (each item measured only one ability) and approximate simple structure (items primarily measured one ability each) were employed for the two-dimensional exam. Let $\beta_{i}$ be the angle between the direction of item $i$ and the axis $\theta_{1}$ defined in Figure 4 . Then, the 


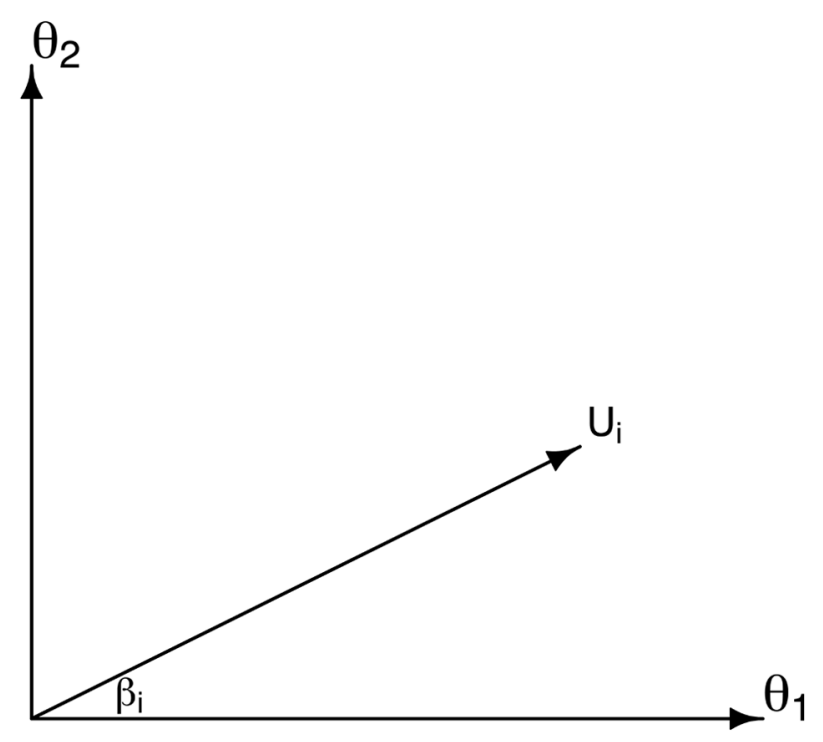

Figure 4. Definition of $\beta_{i}$.

discrimination parameters for item $i$ are decided by $a_{i 1}=a_{i} \cos \beta_{i}$ and $a_{i 2}=a_{i} \sin \beta_{i}$, where $a_{i}$ follows the distribution in Table 1 for $i=1,2, \ldots, n$. For simple structure, $\beta_{i}=0$ for AT items measuring $\Theta_{1}$ only, and $\beta_{i}=\frac{\pi}{2}$ for PT items measuring $\Theta_{2}$ only; for approximate structure, $\beta_{i}$ was randomly selected from uniform $\left[0, \frac{\pi}{9}\right]$ for AT items measuring $\Theta_{1}$ more than $\Theta_{2}$, and $\beta_{i}$ was randomly selected from uniform $\left[\frac{7}{18} \pi, \frac{\pi}{2}\right]$ for PT items measuring $\Theta_{2}$ more than $\Theta_{1}$.

As with the Type I error study, both a moderately long test (52 points) and a medium length test (32 points) were employed. To study the effect of MC-CR composition, four mixtures of $\mathrm{MC}$ and CR items were employed: $100 \% \mathrm{MC}$ items, $85 \% / 15 \% \mathrm{MC} / \mathrm{CR}, 50 \% / 50 \% \mathrm{MC} / \mathrm{CR}$, and $100 \%$ CR. Different item partitions were used to simulate two-dimensional item response data where the smaller subtest measured $\Theta_{1}$ more, denoted as $A_{1}$, and the larger subtest measured $\Theta_{2}$ more, denoted as $A_{2}$. For the 52-point test, $A_{1}$ consisted of five or $10 \mathrm{MC}$ items, or two or five $\mathrm{CR}$ items, or 10 mixed items $(5 \mathrm{MC}+5 \mathrm{CR})$. For the 32-point test, $A_{1}$ consisted of either three or six MC items, or simply three CR items, or six mixed items $(3 \mathrm{MC}+3 \mathrm{CR})$. The number of exams and $A_{1}$ combinations is 25 (see Table 3). To study the effect of sample size, the number of simulated examinees was 500 or 1,000 . The effect of the true item partition for both simple structured and approximate simple structured two-dimensional tests was investigated. To better evaluate the three AT-selection methods, the perfect/true AT selection using $A_{1}$ as the AT subtest directly is also included. The total number of different combinations of factors was $25 \times 2$ sample sizes $\times 3$ correlations $\times 2$ data structures $=300$ for $\mathrm{HCD}, \mathrm{HCP}, \mathrm{HCN}$, and perfect AT selection, respectively.

For dichotomous item exams, Froelich and Habing (2008) used the responses of one third of the examinees in the HCD procedure to select the proper AT subtest, and the rest calculating the DIMTEST without AT2 test statistic. In this study, the authors of the present study investigated whether this partition is still optimal, or if there is a better one for a polytomous exam. Three percentages were compared: $30 \%, 50 \%$, and $70 \%$. For this part of the study, the exam was simulated with simple structure and correlation .7. The examinees used for AT selection were first randomly chosen from the simulated dataset. Second, the AT-selection methods (HCD, HCP, 
Table 3. Simulation Design for Power Study.

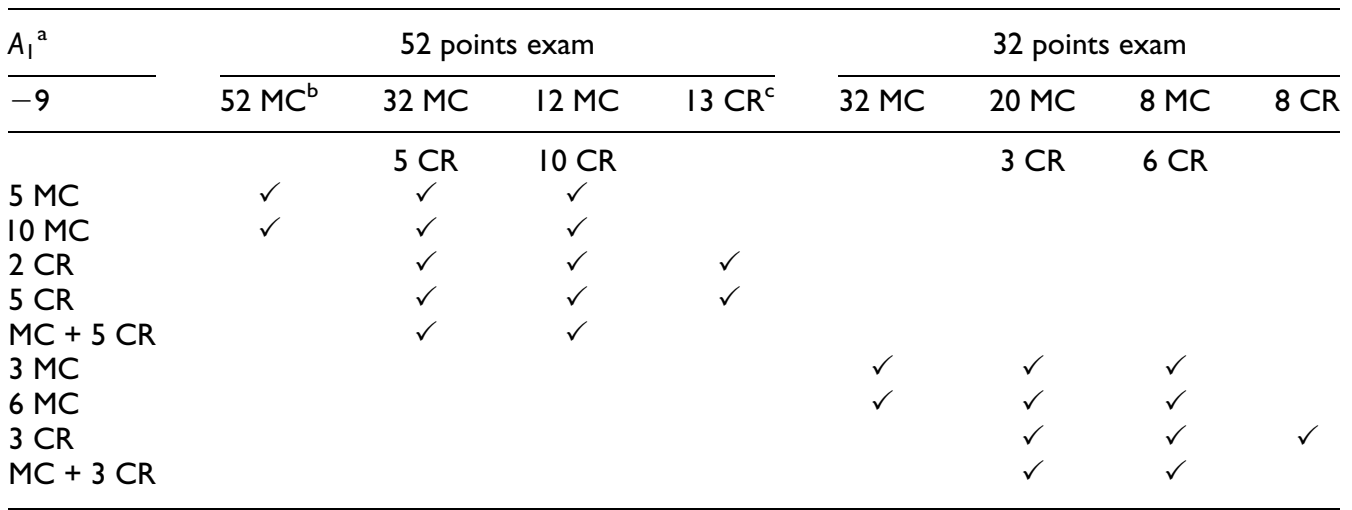

Note. $\mathrm{MC}=$ multiple choice; $\mathrm{CR}=$ constructed response.

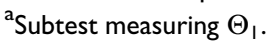

bultiple-choice item.

${ }^{\mathrm{c} C o n s t r u c t e d}$ response item.

and $\mathrm{HCN}$ ) were performed on the selected data to decide the optimal AT/PT pair. Finally, PolyNEWDIM was performed to the rest of the data with the optimal AT/PT pair. The total number of different combinations of factors for each method was 2 exams $\times 4$ compositions $\times 2$ sample sizes $\times 3$ AT-selection methods $=48$ for the Type I error study, and 25 exams and $A_{1}$ combinations $\times 2$ sample sizes $\times 3$ AT percentages $\times 3$ AT-selection methods $=450$ for the power study.

The evaluation criterion was the percent rejection rate based on a significance level of .05 , as recorded more than 400 independent simulation trials to balance run time with accuracy. The standard two-sample test for proportions was used to compare the Type I error rate and power for each combination between every pair of the three AT-selection methods. And the independent samples $t$ test was used to compare Type I error rate and power for all combinations between every pair of the three AT-selection methods. The Bonferroni correction was used to adjust the level of significance for multiple comparisons. The level of significance was $.05 / 3=.017$ after the adjustment.

\section{Appropriate Percentage for AT Selection}

Type I error study: The results indicated that changing percentage of data used for AT selection does not have obvious impact on Type I errors for all of the three AT-selection methods, as all of Type I errors were not significantly changed.

Power study: Figure 5 shows the mean and 95\% confidence interval (CI) of power rate for 25 exams and $A_{1}$ combinations in Table 3 . The figure indicated higher power rate for all of the three AT selection methods when we used more examinees for AT selection (1,000 vs. 500), but not statistically significantly higher based on the independent sample $t$ test. In particular, with sample size 500, 30 of 75 cases over three AT-selection methods showed significant differences in power rates between $30 \%$ and $50 \%$, and 26 cases showed significant differences in power rates between $30 \%$ and $70 \%$, while all of the significant differences were in favor of $50 \%$ or $70 \%$; four cases showed significant differences in power rates between $50 \%$ and $70 \%$, two of 


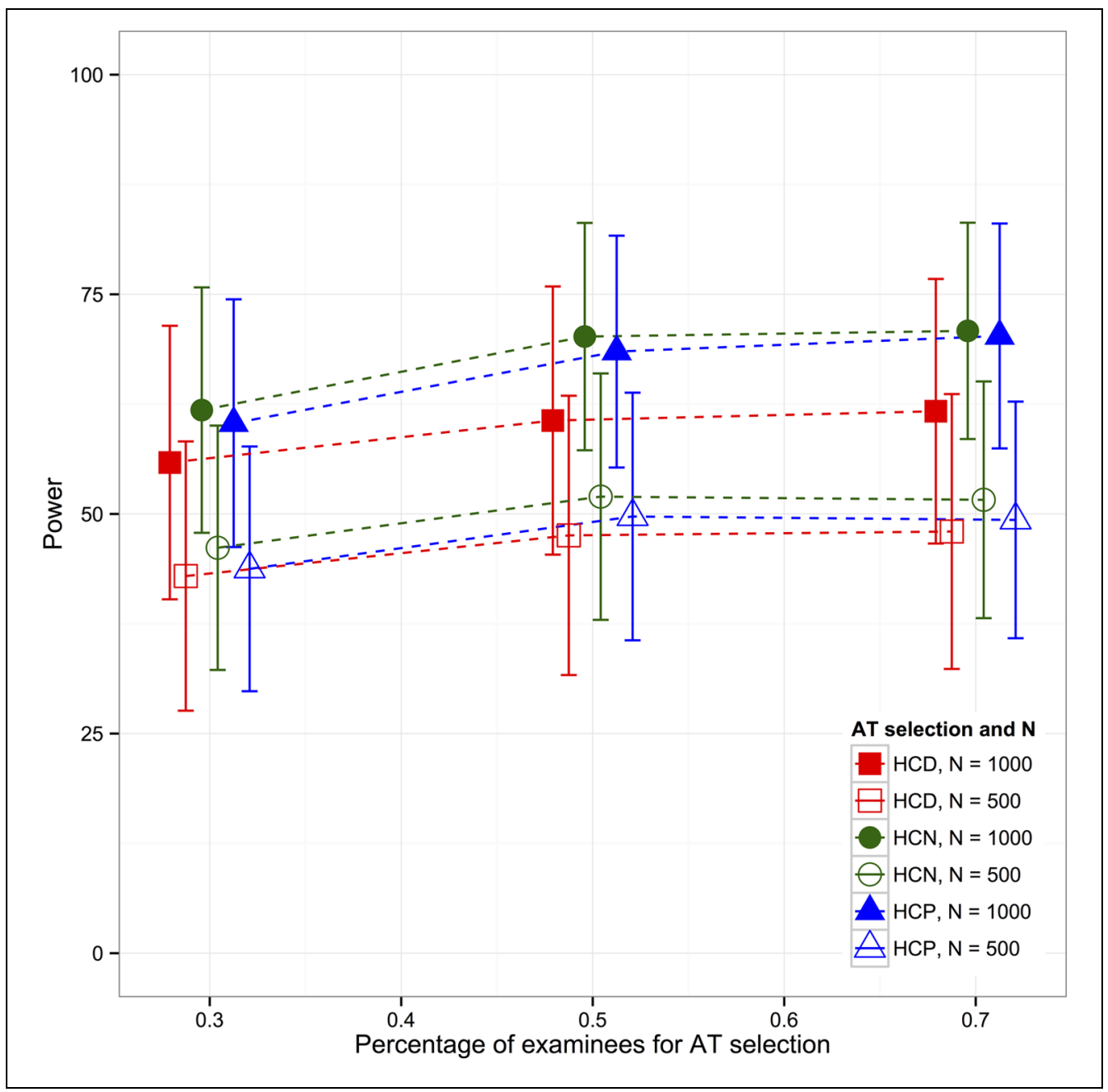

Figure 5. The effect of \% examinees for AT-selection methods on power.

Note. $\mathrm{AT}$ = assessment subtest; HCD = HCA/CCPROX-DETECT; HCN = HCA/CCPROX-PolyNEWDIM; HCP = HCA/CCPROX-PolyDIMTEST.

which were in favor of $70 \%$ and had dichotomous items only in the test. With sample size $1,000,30 \%$ still performed worst among three percentages, six of 75 cases showed significant differences between $50 \%$ and $70 \%$, and were in favor of $70 \%$. Note that .017 was used as the significance level for all the comparisons using the proportion test after Bonferroni correction.

In summary of the simulation results, $30 \%$ is not an appropriate percentage for polytomous version of DIMTEST. Instead, 50\% is appropriate for the test including polytomous items with small sample size such as 500 , while $70 \%$ is good for dichotomous test with small sample size, and $70 \%$ is good for both kinds of tests with large sample size such as 1,000 . The following simulation studies used the appropriate percentages according to this finding. The detailed results for all combinations are shown in Tables 1 to 3 in the online supplement. 
Table 4. Type I Error Results for Three AT-Selection Methods.

\begin{tabular}{|c|c|c|c|c|c|c|c|}
\hline \multirow{2}{*}{$\frac{\text { Test }}{\text { Length }}$} & \multirow{2}{*}{$\begin{array}{c}\text { Test } \\
\text { Form }\end{array}$} & \multicolumn{3}{|c|}{500 examinees } & \multicolumn{3}{|c|}{$\mathbf{I}, 000$ examinees } \\
\hline & & $\mathrm{HCD}^{\mathrm{a}}$ & $\mathrm{HCP}^{\mathrm{b}}$ & $\mathrm{HCN}^{c}$ & $\overline{\mathrm{HCD}}$ & $\mathrm{HCP}$ & $\overline{\mathrm{HCN}}$ \\
\hline & $52 M C$ & 5.25 & 3.75 & 5.25 & 5.50 & 4.50 & 5.00 \\
\hline & $32 M C+5 C R$ & 2.50 & 5.25 & 3.75 & 3.75 & 4.50 & 4.75 \\
\hline \multirow[t]{4}{*}{52 points } & $12 M C+10 C R$ & 1.75 & 3.25 & 4.50 & 2.00 & 2.50 & 2.00 \\
\hline & $13 \mathrm{CR}$ & 2.50 & 2.25 & 2.25 & 2.75 & 2.50 & 2.75 \\
\hline & $32 M C$ & 5.25 & 5.25 & 6.75 & 3.25 & 3.50 & 4.00 \\
\hline & $20 M C+3 C R$ & 2.00 & 4.75 & 6.25 & 2.75 & 5.50 & 6.25 \\
\hline \multirow{2}{*}{32 points } & $8 M C+6 C R$ & 2.00 & 2.25 & 1.75 & 4.25 & 2.25 & 1.75 \\
\hline & $8 \mathrm{CR}$ & 2.25 & 2.75 & 2.75 & 1.50 & 1.75 & 1.50 \\
\hline
\end{tabular}

Note. Boldfaced values indicate significance at $p<.05$. AT = assessment subtest; $M C=$ multiple choice; $\mathrm{CR}=$ constructed response.

${ }^{\mathrm{a}} \mathrm{HCA} / \mathrm{CCPROX}-\mathrm{DETECT}$.

bHCA/CCPROX-PolyDIMTEST using Poly-DIMTEST without AT2's test statistic.

'HCA/CCPROX-PolyNEWDIM using Poly-NEWDIM's test statistic.

\section{Type I Error Study}

At the .05 significance level, the $95 \%$ confidence range for the rejection rate would be $2.86 \%$ to $7.14 \%$. This range is used to indicate acceptable performance, and rates out of this range (highlighted in Table 4) are statistically significantly different of .05. None of the tested conditions show excessive Type I error rates $(>7.14 \%)$. When the sample size is 500 , HCD was overly conservative with six of eight cases having low Type I error rates $(<2.86 \%)$, whereas three of eight cases for each proposed method (HCP and HCN) did. When the sample size increased to 1,000, however, all three methods gave over conservative Type I error rates for four of eight cases. In addition, most of the overly conservative Type I error rates occurred to the exams with no more than 22 items. Note that there are almost no differences among the three methods for the $13 \mathrm{CR}$ and eight CR exams. The reason is that the HCA/CCPROX procedure provides limited cluster pairs for small numbers of items.

\section{Power Study}

Table 5 shows the power rate (\%) for AT-selection methods for simple structured data with 500 examinees and a correlation of .6. The power rates higher than $80 \%$ (highlighted in the table) are considered as adequate power for Poly-NEWDIM. The perfect AT selection (using $A_{1}$ as the AT subtest) shows good power for most cases, except for four cases of short AT subtest (five dichotomous items). It indicates that Poly-NEWDIM does not work well with short true AT subtest, especially when the test is short (three of four cases were from 32 points test). It is not surprising that three AT-selection methods performed poorly when true AT subtest $\left(A_{1}\right)$ is short (five dichotomous items). The power rate increases for longer true AT subset. While three methods show good power rates for the same number of cases (10 of 25), HCP and HCN show significantly higher power than HCD for most of the rest of the cases with dichotomous true AT subtest based on the two-sample proportion test with Bonferroni correction ( $p$ values $<$.017). HCP performed worse than the other two for polytomous AT subtests. The other combinations of correlation, sample size, and data structure show similar patterns. Detailed results are provided in Tables 4 to 9 in the online supplement. In total, more than 80 of 300 cases for three methods ( 83 for $\mathrm{HCD}, 87$ for $\mathrm{HCP}$, and 88 for $\mathrm{HCN}$ ) show power higher than $80 \%$. 
Table 5. The Power Rate (\%) for AT-Selection Methods With Correlation .6, 500 Examinees, and Simple Structure.

\begin{tabular}{|c|c|c|c|c|c|c|}
\hline Test length & Test form & $A_{1}{ }^{\mathrm{a}}$ & $\mathrm{HCD}$ & HCP & $\mathrm{HCN}$ & Perfect $A T^{\mathrm{b}}$ \\
\hline & $52 M C$ & $5 M C$ & 10.00 & $19.50^{c}$ & $13.75^{d}$ & 77.50 \\
\hline & & $10 \mathrm{MC}$ & 49.00 & $70.25^{c}$ & $63.00^{\mathrm{cd}}$ & 99.00 \\
\hline & & $5 \mathrm{MC}$ & 7.00 & $22.25^{c}$ & $18.75^{c}$ & 88.00 \\
\hline & $32 \mathrm{MC}+$ & $10 \mathrm{MC}$ & 50.25 & $79.75^{c}$ & $73.50^{\text {cd }}$ & 99.75 \\
\hline & $5 \mathrm{CR}$ & $2 \mathrm{CR}$ & 44.25 & $21.50^{c}$ & $39.25^{d}$ & 96.50 \\
\hline & & $5 C R$ & 99.75 & 95.25 & 95.75 & 100.00 \\
\hline & & $5 M C+5 C R$ & 99.75 & 97.50 & 98.25 & 100.00 \\
\hline \multirow[t]{12}{*}{52 points } & & $5 M C$ & 8.75 & $24.25^{c}$ & $27.5^{c}$ & 86.50 \\
\hline & $12 M C+$ & $10 M C$ & 61.50 & $80.25^{c}$ & $82.75^{c}$ & 99.75 \\
\hline & $10 \mathrm{CR}$ & $2 C R$ & 47.25 & $35.00^{c}$ & $53.25^{d}$ & 93.25 \\
\hline & & $5 \mathrm{CR}$ & 99.50 & 99.25 & 98.75 & 100.00 \\
\hline & & $5 M C+5 C R$ & 100.00 & 100.00 & 100.00 & 100.00 \\
\hline & $13 C R$ & $2 C R$ & 53.25 & $63.50^{c}$ & $67.00^{c}$ & 95.25 \\
\hline & & $5 C R$ & 100.00 & 99.75 & 100.00 & 100.00 \\
\hline & $32 M C$ & $3 \mathrm{MC}$ & 6.75 & $12.75^{c}$ & 9.75 & 43.50 \\
\hline & & $6 M C$ & 23.25 & $44.50^{c}$ & $39.75^{c}$ & 84.25 \\
\hline & & $3 M C$ & 5.50 & $9.75^{c}$ & 8.25 & 53.50 \\
\hline & $20 \mathrm{MC}+$ & $6 \mathrm{MC}$ & 30.00 & $54.25^{c}$ & $46.50^{\text {cd }}$ & 94.25 \\
\hline & $3 \mathrm{CR}$ & $3 C R$ & 91.00 & $72.00^{c}$ & $78.25^{\mathrm{cd}}$ & 99.50 \\
\hline \multirow[t]{6}{*}{32 points } & & $3 M C+3 C R$ & 94.50 & 89.00 & 89.00 & 99.75 \\
\hline & & $3 M C$ & 6.00 & $12.75^{c}$ & $13.75^{c}$ & 58.00 \\
\hline & $8 M C+$ & $6 \mathrm{MC}$ & 44.75 & $58.25^{c}$ & $60.50^{c}$ & 91.75 \\
\hline & $6 \mathrm{CR}$ & $3 C R$ & 95.00 & 94.25 & 96.00 & 100.00 \\
\hline & & $3 M C+3 C R$ & 97.00 & 98.00 & 97.50 & 99.75 \\
\hline & $8 \mathrm{CR}$ & $3 \mathrm{CR}$ & 99.50 & 97.00 & 96.75 & 100.00 \\
\hline
\end{tabular}

Note. $\mathrm{AT}=$ assessment subtest; $\mathrm{HCD}=\mathrm{HCA} / \mathrm{CCPROX}$-DETECT; HCP = HCA/CCPROX-PolyDIMTEST; HCN = HCA/CCPROX-PolyNEWDIM; $M C=$ multiple choice; $C R=$ constructed response.

${ }^{a}$ Subtest measuring $\Theta_{1}$.

b Use $A_{1}$ as AT.

'Significantly different from HCD at $p<.017$.

${ }^{\mathrm{d}}$ Significantly different from HCP at $p<.017$.

To visualize the overall pattern across various simulation conditions, figures of marginal means of power rates are created. Figure 6 shows the marginal means and 95\% CIs of power rates for three AT-selection methods at different correlations, .6, .7, and .8 over a total of 100 cases $(25$ combinations in Table $3 \times 2$ sample sizes $\times 2$ data structures). Among the three ATselection methods, HCN provided highest power on average, and HCD provided lowest power, at each correlation. The differences were not statistically significant based on the independent samples $t$ test with Bonferroni correction. The power dropped significantly for all three methods when the correlation between the two latent abilities increased from .6 to .8 based on the proportion test with Bonferroni correction. According to Tables 4 to 9 in the online supplement, which shows the detailed results for all 100 cases, the power rates of HCN decreased significantly less than HCP for 11 of 100 cases, while the power rates of $\mathrm{HCN}$ decreased significantly more than HCP for seven cases. This finding implied that the power of $\mathrm{HCN}$ is less sensitive to high correlation among latent abilities in more situations.

Figure 7 shows the effect of sample size on power rates. It indicates that $\mathrm{HCN}$ still provided highest power on average of the 150 cases $(25$ combinations in Table $3 \times 2$ sample sizes $\times 3$ 


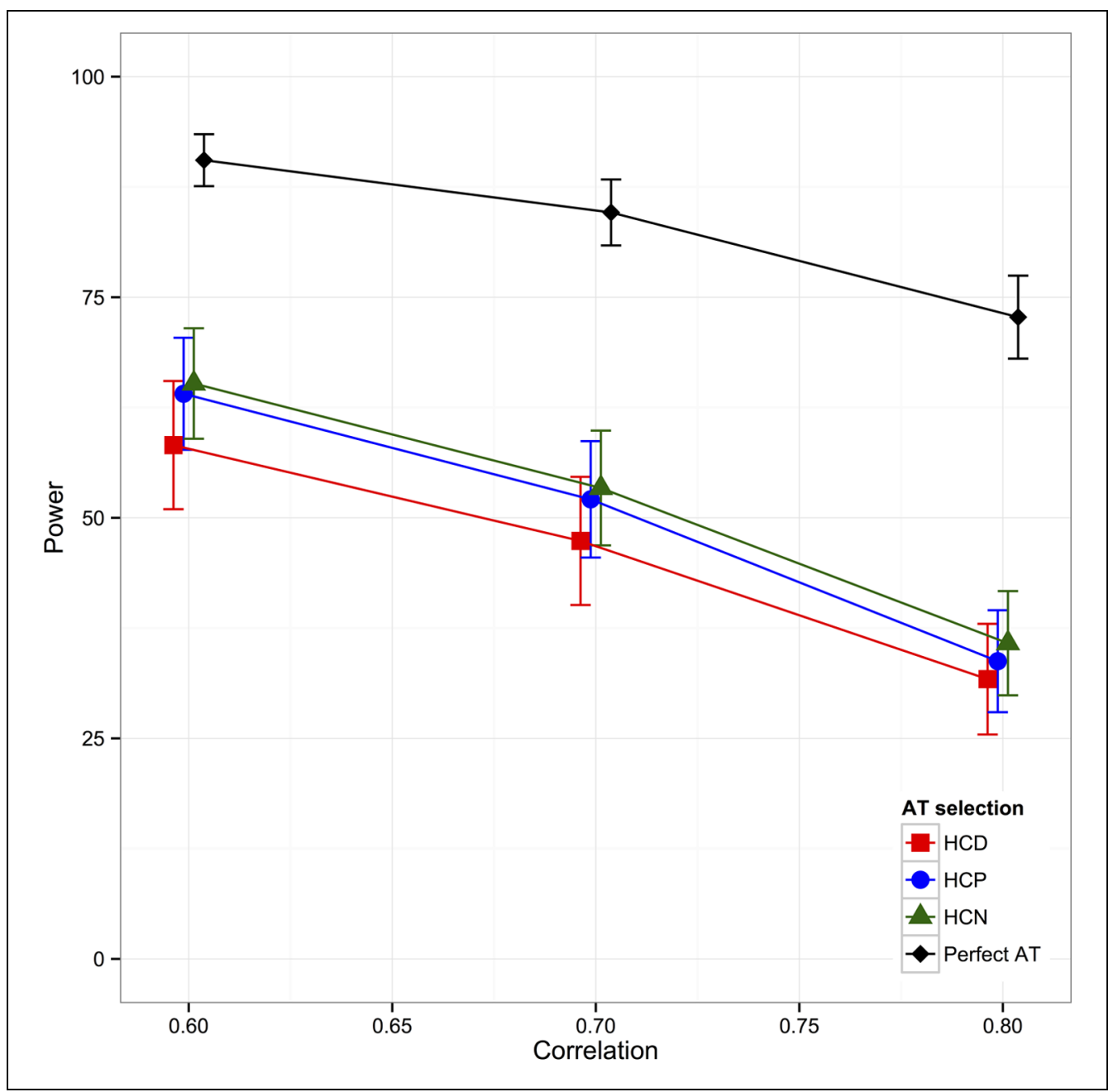

Figure 6. The effect of correlation on power rate for AT-selection methods.

Note. $\mathrm{AT}=$ assessment subtest; HCD = HCA/CCPROX-DETECT; HCP = HCA/CCPROX-PolyDIMTEST; HCN = HCA/CCPROX-PolyNEWDIM.

correlations), and HCD provided lowest power at each sample size (500 and 1,000). Again, the differences were not statistically significant based on the independent samples $t$ test with Bonferroni correction. The power increased significantly for the three methods when the sample size increased from 500 to 1,000 based on the proportion test with Bonferroni correction. In detail, according to Tables 4 to 9 in the online supplement, when the sample size dropped from 1,000 to 500 , for 13 of 150 cases the power rates decreased significantly less for HCN than for $\mathrm{HCP}$, while for six cases, the power rates decreased significantly more for HCN than for HCP. Thus, $\mathrm{HCN}$ is less sensitive to small sample size.

Figure 8 shows the effect of data structure. It indicates a similar pattern for two data structures, simple structure versus approximate simple structure. HCN performed best and HCD performed worst at each data structure, although the difference was not statistically significant based on the independent samples $t$ test with Bonferroni correction. The power decreased 


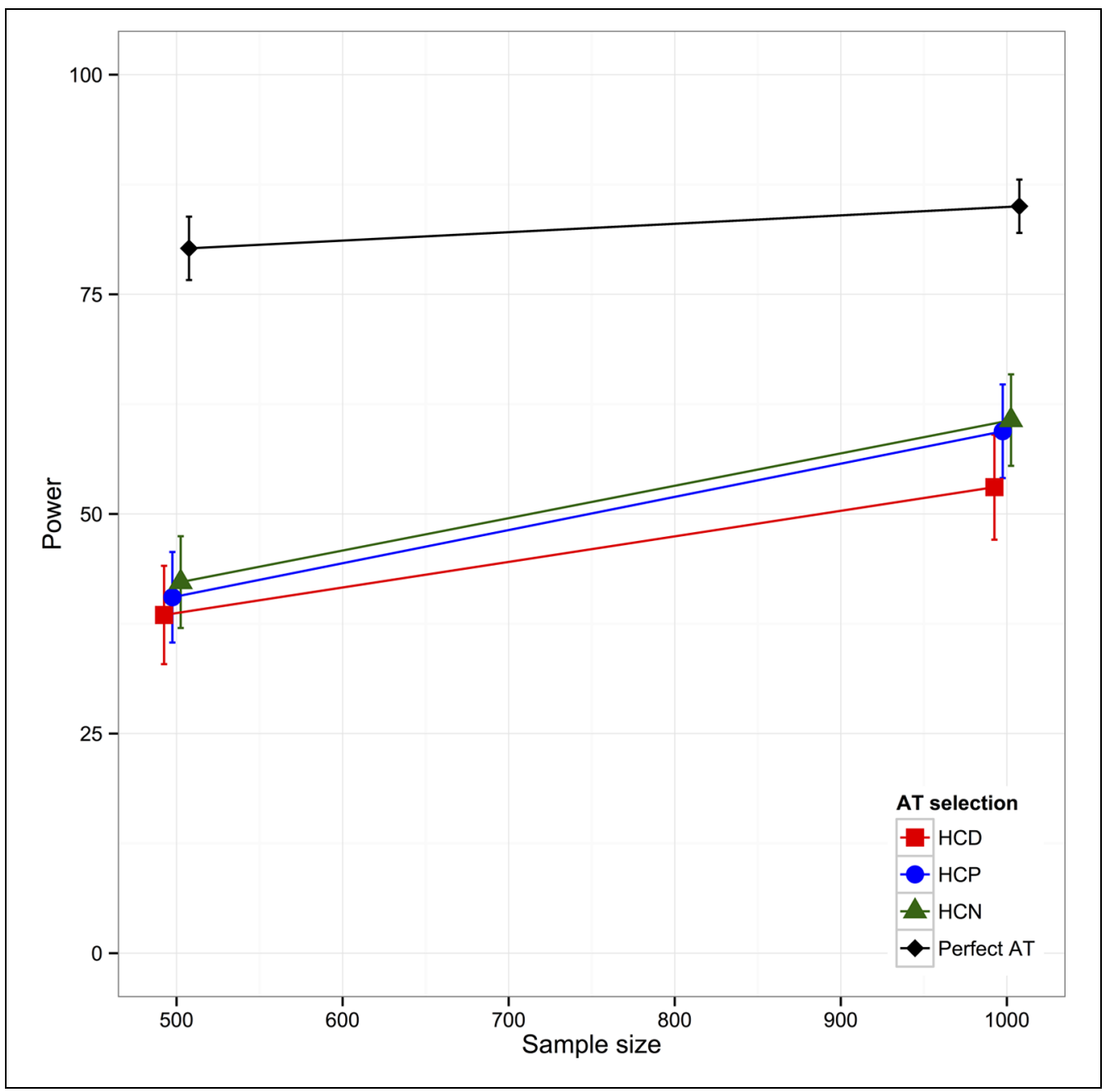

Figure 7. The effect of sample size on power rate for AT-selection methods. Note. $\mathrm{AT}=$ assessment subtest; $\mathrm{HCD}=\mathrm{HCA} / C C P R O X-D E T E C T ; \mathrm{HCP}=\mathrm{HCA} / C C P R O X-P o l y D I M T E S T ; H C N=$ HCA/CCPROX-PolyNEWDIM.

significantly for the three methods when the data structure changed from simple to approximate simple structure based on the proportion test with Bonferroni correction. In addition in detail, for 13 of all 150 cases the power rates decreased significantly less for $\mathrm{HCN}$ than for HCP, whereas for 10 cases the power rates decreased significantly more for $\mathrm{HCN}$ than for HCP. Thus, HCN is less sensitive to complexity of the data structure.

\section{Concluding Remarks}

Poly-NEWDIM (Li et al., 2010; Li, 2011), an improved polytomous version of DIMTEST, employs the nonparametric IRT-based parametric bootstrap procedure as the bias correction method. It results in good power when AT and PT are correctly selected. However, the decision of AT and PT is vital to the performance of the Poly-NEWDIM procedure. Two new 


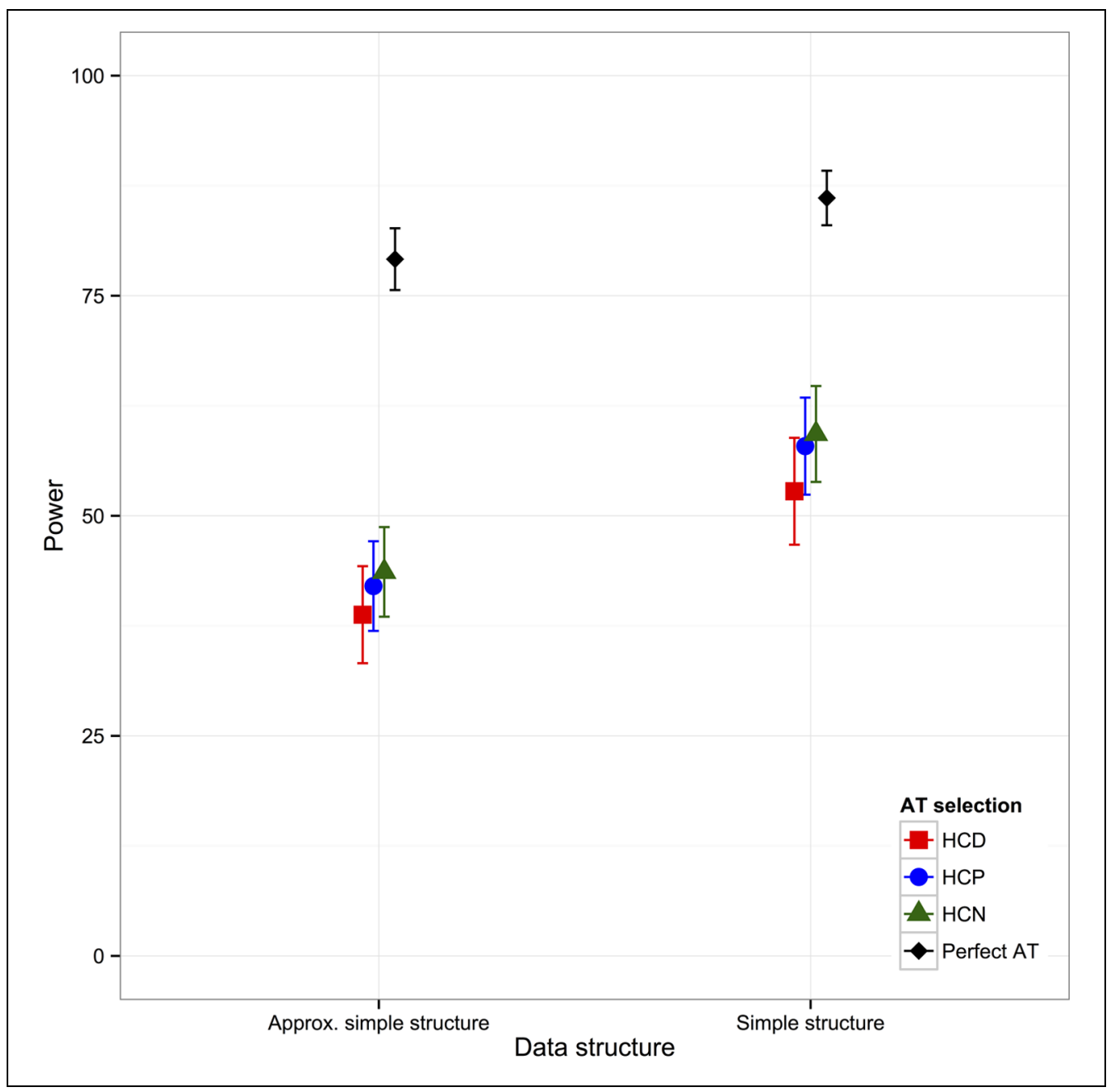

Figure 8. The effect of data structure on power rate for AT-selection methods.

Note. $\mathrm{AT}=$ assessment subtest; HCD = HCA/CCPROX-DETECT; HCP = HCA/CCPROX-PolyDIMTEST; HCN = HCA/CCPROX-PolyNEWDIM.

AT-selection procedures, $\mathrm{HCP}$ and $\mathrm{HCN}$, are proposed based on the test statistic of PolyDIMTEST without AT2 and Poly-NEWDIM, respectively.

Generally, the three methods performed closely in the Type I error study, while HCN performed better than HCD and HCP in the power study for most tested cases. The results showed that $\mathrm{HCN}$ improves the performance of Poly-NEWDIM significantly when power is lower than $80 \%$, and performed better or equally when power is higher than $80 \%$. It however still lacks the power obtained with perfect AT selection as shown in Figures 6 to 8. Furthermore, the power increased with increasing length of $A_{1}$ and sample size, and decreased with increasing correlation and additional structural complexity. HCN showed less sensitivity than HCP to sample size, correlation, and structural complexity. Note that the methods in this study showed conservative Type I error rates for short exams ( $\leq 22$ items), which may lead to decreased power. Both methods still resulted in poor power rates when $A_{1}$ includes no more than five dichotomous items even with low correlation (.6), which is not surprising because of the poor power provided by 
the perfect AT selection for short exams. Therefore, further study will be needed to see if polytomous DIMTEST can be improved when the exam and $A_{1}$ are short. For long exams, the lowpower cases also happen when $A_{1}$ is short. This is probably because HCA/CCPROX algorithm is not able to pick up the correct cluster for small AT. In the future, other clustering algorithms such as brute-force enumeration for this kind of situation will be explored.

The appropriate percentage of examinees used for AT selection was also investigated by simulation study. The results showed that using $70 \%$ of the examinees for AT selection and $30 \%$ for poly-NEWDIM is appropriate for all three kinds of test (dichotomous only, polytomous only, and mixed) with large sample size and dichotomous exams with small sample size, while $50 \% / 50 \%$ is good for polytomous exams and mixed exams with small sample size.

In summary, the AT-selection method, $\mathrm{HCN}$, proposed in this study demonstrated good performance in terms of Type I error and power rates when used for a long exam ( $>22$ items) with not too small subtest ( $<$ five dichotomous items or two polytomous items) measuring one of the latent abilities. The power was up to $20 \%$ larger than that obtained from the previous method in some simulation settings.

The simulation studies in this study focused on simple and approximate simple structured two-dimensional exams. More complex data structures, such as fan structures, and more dimensions (three or four) also require future study. The performance of the authors' method on real data in the future will also be evaluated.

\section{Declaration of Conflicting Interests}

The author(s) declared no potential conflicts of interest with respect to the research, authorship, and/or publication of this article.

\section{Funding}

The author(s) received no financial support for the research, authorship, and/or publication of this article.

\section{References}

Ackerman, T. A. (1989). Unidimensional IRT calibration of compensatory and noncompensatory multidimensional items. Applied Psychological Measurements, 13, 113-127.

Ackerman, T. A. (1996). Graphical representation of multidimensional item response theory. Applied Psychological Measurement, 20, 311-329.

Birnbaum, A. (1968). Some latent trait models and their use in inferring an examinee's ability. In F. M. Lord \& M. R. Novick (Eds.), Statistical theories of mental test scores (pp. 397-472). Reading, MA: Addison-Wesley.

Froelich, A., \& Habing, B. (2008). Conditional covariance-based subtest selection for DIMTEST. Applied Psychological Measurement, 32, 138-155.

Habing, B. (2001). Nonparametric regression and the parametric bootstrap for local dependence assessment. Applied Psychological Measurement, 25, 221-233.

Hattie, J. A. (1985). Methodology review: Assessing unidimensionality of tests and items. Applied Psychological Measurement, 9, 139-164.

Hattie, J. A., Krakowski, K., Rogers, H. J., \& Swaminathan, H. (1996). An assessment of Stout's index of essential unidimensionality. Applied Psychological Measurement, 20, 1-14.

Kim, H. R. (1994). New techniques for the dimensionality assessment of standardized test data (Unpublished doctoral dissertation). University of Illinois at Urbana-Champaign, Department of Statistics, Champaign.

Kirisci, L., \& Hsu, T. (1995, April). The robustness of BILOG to violations of the assumptions of unidimensionality of test items and normality of ability distribution. Paper presented at the annual meeting of the National Council on Measurement in Education, San Francisco, CA. 
Li, H.-H., \& Stout, W. F. (1995, April). Assessment of unidimensionality for mixed polytomous and dichotomous item data: Refinements of Poly-DIMTEST. Paper presented at the Annual Meeting of the National Council on Measurement in Education, San Francisco, CA.

Li, T. (2011). Assessing the dimensionality of polytomous item exams (Doctoral dissertation). Retrieved from http://scholarcommons.sc.edu/etd/2672

Li, T., Habing, B., \& Roussos, L. (2010, May). Conditional covariance-based subtest selection for polytomous item DIMTEST. Paper presented at the annual meeting of the National Council on Measurement in Education, Denver, CO

Li, T., Habing, B., \& Roussos, L. (2011, April). Improved conditional covariance-based subtest selection for polytomous DIMTEST. Paper presented at the annual meeting of the American Educational Research Association (AERA), New Orleans, LA.

Nandakumar, R., Yu, F., Li, H., \& Stout, W. (1998). Assessing unidimensionality of polytomous data. Applied Psychological Measurement, 22, 99-115.

Reckase, M. D. (1985). The difficulty of test items that measure more than one ability. Applied Psychological Measurement, 9, 401-412.

Reckase, M. D. (1997). A linear logistic multidimensional model for dichotomous item response data. In W. J. van der Linden \& R. K. Hambleton (Eds.), Handbook of modern item response theory (pp. 271286). New York, NY: Springer.

Reckase, M. D. (2009). Multidimensional Item Response Theory (Statistics for social and behavioral sciences). New York, NY: Springer.

Reckase, M. D., \& McKinley, R. L. (1991). The discriminating power of items that measure more than one dimension. Applied Psychological Measurement, 15, 361-373.

Roussos, L. A., Stout, W. F., \& Marden, J. I. (1998). Using new proximity measures with hierarchical cluster analysis to detect multidimensionality. Journal of Educational Measurement, 35, 1-30.

Samejima, F. (1997). Graded response model. In W. J. van der Linden \& R. K. Hambleton (Eds.), Handbook of modern item response theory (pp. 85-100). New York, NY: Springer.

Seo, M., \& Roussos, L. A. (2009, April). Evaluation of DIMTEST effect-size measure and its application. Paper presented at the Annual Meeting of the National Council on Measurement in Education, San Diego, CA.

Seo, M., \& Roussos, L. A. (2010). Formulation of a DIMTEST effect size measure (DESM) and evaluation of the DESM estimator bias. Journal of Educational Measurement, 47, 413-431.

Seraphine, A. E. (2000). The performance of DIMTEST when latent trait and item difficulty distributions differ. Applied Psychological Measurement, 42, 82-94.

Stout, W. F. (1987). A nonparametric approach for assessing latent trait unidimensionality. Psychometrika, $52,589-617$.

Stout, W. F., Froelich, A. G., \& Gao, F. (2001). Using resampling methods to produce an improved DIMTEST procedure. In A. Boomsma, M. A. J. van Duijn, \& T. A. B. Snijders (Eds.), Essays on item response theory (pp. 357-375). New York, NY: Springer.

Stout, W. F., Habing, B., Douglas, J., Kim, H. R., Roussos, L., \& Zhang, J. (1996). Conditional covariance-based nonparametric multidimensionality assessment. Applied Psychological Measurement, 19, 331-354.

Tate, R. (2003). A comparison of selected empirical methods for assessing the structure of responses to test items. Applied Psychological Measurement, 27, 159-203.

Zhang, J. (2007). Conditional covariance theory and DETECT for polytomous items. Psychometrika, 72, 69-91.

Zhang, J., \& Stout, W. F. (1999a). Conditional covariance structure of generalized compensatory multidimensional items. Psychometrika, 64, 129-152.

Zhang, J., \& Stout, W. F. (1999b). The theoretical DETECT index of dimensionality and its application to approximate simple structure. Psychometrika, 64, 213-219. 